\title{
ANÁLISIS DEL SISTEMA DE VIGILANCIA CENTINELA DE LAS INFECCIONES RESPIRATORIAS AGUDAS GRAVES, HOSPITAL INFANTIL DR. ROBERT REID CABRAL, REPÚBLICA DOMINICANA, 2015-2019 \\ Analysis of the sentinel surveillance system of Severe Acute Respiratory
Infections, Dr. Robert Reid Cabral Children's Hospital, Dominican Republic, 2015-2019
}

\author{
Pablo Mancebo García ${ }^{a}$, Feliezer Romano ${ }^{b}$, Janahira Sánchezc ${ }^{c}$ Delia Castillo ${ }^{d}$, \\ Lorennys María y Elizabeth Valdez
}

Recibido: 23 de marzo, 2020 • Aprobado: 6 de julio, 2020

Cómo citar: Mancebo García P, Romano F, Sánchez J, Castillo D, María L, Valdez E. Análisis del sistema de vigilancia centinela de las Infecciones Respiratorias Agudas Graves, Hospital Infantil Dr. Robert Reid Cabral, República Dominicana, 2015-2019. cysa [Internet]. 15 de febrero de 2021 [citado 23 de febrero de 2021];5(1):57-. Disponible en: https://revistas.intec.edu.do/index.php/cisa/article/ view/2062

\section{Resumen}

Introducción: la vigilancia centinela de Infecciones Respiratorias Agudas Graves (IRAG) inició en República Dominicana en febrero del año 2008, en dos hospitales: el Hospital Infantil Dr. Robert Reid Cabral (HIRRC) en Santo Domingo y el Hospital José María Cabral y Báez, en Santiago. Este estudio tiene como objetivo general describir el comportamiento de las infecciones respiratorias agudas graves (IRAG) captadas en el Hospital Infantil Dr. Robert Reid Cabral durante los ańos 2015-2019.

Método: se realizó un estudio observacional, descriptivo, de corte transversal, con recolección retrospectiva de datos, de los casos de IRAG reportados en el puesto centinela del Hospital Infantil de Referencia Nacional Dr. Robert Reid Cabral, Área V de salud, Distrito Nacional, desde el año 2015 hasta el año 2019. Se hace una descripción de las características de los casos ingresados a la base de datos del sistema, los virus respiratorios identificados, comportamiento en el tiempo, persona y lugar.

\footnotetext{
a Departamento de Epidemiología. Hospital Infantil Dr. Robert Reid Cabral. Santo Domingo, Republica Dominicana.

Correo-e: epidemiologia@hirrc.gov.do

b Departamento de Epidemiologia. Hospital Infantil Dr. Robert Reid Cabral. Correo-e: feliezer_21@hotmail.com

${ }^{\mathrm{c}}$ Departamento de Epidemiologia. Hospital Infantil Dr. Robert Reid Cabral. Correo-e: janahira33@gmail.com
}

\begin{abstract}
Introduction: Sentinel surveillance of Severe Acute Respiratory Infections (SARI) began in the Dominican Republic in February 2008 in two hospitals: Dr. Robert Reid Cabral Children's Hospital (HIRRC) in Santo Domingo and José María Cabral y Báez Hospital, in Santiago. The purpose of this study is to describe the behavior of severe acute respiratory infections (SARI) caught at the Dr. Robert Reid Cabral Children's Hospital during the years 2015-2019.
\end{abstract}

Method: An observational, descriptive, cross-sectional study was carried out, with retrospective data collection, of SARI cases reported in the sentinel post of the Dr. Robert Reid Cabral Children's National Reference Hospital, from the year 2015 to the year 2019. A description is made of the characteristics of the cases entered into the system database, the respiratory viruses identified, behavior over time, person and place.

\footnotetext{
d Departamento de Epidemiologia. Hospital Infantil Dr. Robert Reid Cabral. Correo-e: delia.castillo@ministeriodesalud.gob.do

${ }^{\mathrm{f}}$ Departamento de Epidemiologia. Hospital Infantil Dr. Robert Reid Cabral. Correo-e: Lorennys-28@hotmail.com

e Departamento de Epidemiologia. Hospital Infantil Dr. Robert Reid Cabral. Correo-e: eliusmic23@gmail.com
} 
Resultados: la captación de infecciones respiratorias agudas graves (IRAG) a través del puesto centinela las IRAG, representaron $14.37,10.13,4.10,3.70$ y $3.42 \%$ de las IRA, respectivamente, en los últimos cinco años. En las semanas epidemiológicas, en el año 2015 hubo una mayor cantidad de casos entre las semanas 9-16 y 40-48, con un pico alto en la semana 47, en el año 2016 estuvo concentrado entre las semanas 24-42, en el año 2017, en las semanas 33-38, en el ańo 2018 se presentaron con mayor frecuencia entre las semanas $10-15$ y con altas y bajas entre la semana 25-49, y ya para el año 2019 se reportaron casos de manera irregular con altas y baja durante la semana 6-41. La detección de la circulación del Virus Sincitial Respiratorio (VSR) se identificó puntualmente con mayor porcentaje en los años 2015, 2016, 2017 y el año 2019 en comparación con el año 2018 donde el agente predominante fue el virus de la influenza A H1N1. El mayor pico de mortalidad por IRAG estuvo presente en el año 2015 con 18 muertes.

Conclusión: la captación de infecciones respiratorias agudas graves (IRAG) a través del puesto centinela disminuyó en los años 2017 (501 casos), 2018 (465 casos) y 2019 (381 casos), debido, principalmente, a una modificación de la definición de casos establecida por la OPS/OMS y remitida por la Dirección General de Epidemiología, representando solo un 4.10, 3.70 y $3.42 \%$, respectivamente, de las IRA en esos últimos tres años de estudio; sin embargo, en los 2 primeros años de estudio, 2015 (1706 casos) y 2016 (1350 casos), cuando no se habían disminuido los criterios de definición de caso, las IRAG representaron 14.37 y $10.13 \%$ de las IRA, respectivamente.

Palabras clave: vigilancia; centinela; Infección Respiratoria Aguda Grave; morbilidad; pediatría.
Results: The uptake of severe acute respiratory infections (SARI) through the sentinel station SARI, represented $14.37,10.13,4.10,3.70$ and $3.42 \%$ of the ARI respectively, in the last 5 years. In epidemiological weeks, in the year 2015 there were a greater number of cases between weeks 9-16 and 40-48, with a high peak in week 47, in 2016 it was concentrated between weeks 24-42, in 2017, in weeks 33- 38, in 2018 they occurred more frequently between weeks 10-15 and with discharges and losses between week 25-49 and by 2019 irregular cases with discharges and discharges were reported during week 6-41. The Respiratory Syncytial Virus was identified with a higher percentage in 2015, 2016, 2017 and 2019 compared to the year 2018 where the predominant agent was the influenza A H1N1 virus. The highest peak of SARI mortality was present in 2015 with 18 deaths.

Conclusion: The uptake of severe acute respiratory infections (SARI) through the sentinel surveillance decreased in the years 2017 (501 cases), 2018 (465 cases) and 2019 (381 cases), mainly due to a modification of the definition of cases established by the PAHO / WHO and submitted by the General Directorate of Epidemiology, representing only $4.10,3.70$ and $3.42 \%$ respectively of ARI in those last three years of study. However, in the first 2 years of the study, 2015 (1706 cases) and 2016 (1,350 cases), where the case definition criteria had not been decreased, SARIs represented 14.37 and 10.13 percent of ARI respectively.

Keywords: Surveillance; ssentinel; Severe Acute Respiratory Infection; morbidity; pediatrics. 


\section{Introducción}

Las Infecciones Respiratorias Agudas (IRA) constituyen un grupo complejo y heterogéneo de enfermedades ocasionadas por un gran número de agentes causales que afectan las vías respiratorias y representan para todos los países un importante problema de salud por sus grandes cifras de morbilidad y mortalidad, a todo lo cual contribuyen las dificultades inherentes a la implementación de programas eficaces para su prevención y control. ${ }^{1}$ Las IRA se clasifican en altas y bajas según afecten, fundamentalmente, en el sistema respiratorio, estructuras por encima de la laringe o por debajo de esta. A su vez estas se dividen en no complicadas y complicadas, son causadas por distintos gérmenes, que afectan cualquier parte del aparato respiratorio y se describen entidades de acuerdo con el lugar donde predominan los síntomas. ${ }^{2}$

Infección Respiratoria Aguda Grave inusitada o imprevista: corresponde a aquellos casos de IRA que requiere hospitalización (IRAG), con características que lo hacen inusuales y atípicas. ${ }^{3}$ Entre las bacterias que causan estas infecciones se pueden mencionar: Streptococcus pneumoniae, los serotipos 14, 6B, 23F, 1, 5, 6A, 19F, 18B y 9V son los más frecuentes en el país. Haemophilus Influenzae serotipo b, principalmente en niños de 4 meses a 2 años y Staphylococcus spp. Los bacilos Gram negativos (Klebsiella spp. Escherichia coli, etc.), Mycoplasma pneumoniae y Chlamydia pneumoniae, son los siguientes en frecuencia. ${ }^{4}$

Entre el 2017 y el 2019 se reportan alrededor de 2.3 millones de episodios (atenciones) de IRA por año; con índice epidémico dentro de lo esperado. En lo que respecta a la vigilancia centinela de IRAG, en el año 2017 se registraron 1090 casos, mientras que en 2018 y 2019, se reportaron 2108 y 2109 casos, respectivamente. ${ }^{5}$ Se ha estimado que las IRA representan entre el 30 y el $50 \%$ de las visitas para los niños a los establecimientos de salud, y entre el 20 y el $40 \%$ de las hospitalizaciones pediátricas en la mayoría de los países en vías de desarrollo, constituyendo la principal causa de morbilidad en el mundo y la causa más frecuente de utilización de los servicios de salud en todos los países. ${ }^{6}$

Se realizó un estudio descriptivo de los casos de IRAG reportados en el puesto centinela del Hospital Infantil de Referencia Nacional Dr. Robert Reid Cabral -HIRRC-, Área V, Distrito Nacional), desde el año 2015 hasta el año 2019. Se hizo una descripción de las características de los casos ingresados a la base de datos del sistema, los virus respiratorios identificados, comportamiento en el tiempo, persona y lugar. Se realiza un análisis de la base de datos de la plataforma web del Módulo de Vigilancia Especial del Sistema Nacional de Vigilancia Epidemiológica (SINAVE).

En la actualidad, el COVID-19 ha estado afectando a una comunidad considerable en diferentes países, especialmente en los países asiáticos, extendiéndose a Europa, Estados Unidos y Australia. Según cifras del Banco Central, la República Dominicana recibe un importante flujo de viajeros anualmente procedentes de los cinco continentes, cifra que llegó a 17, 126,857 en 2019, de los cuales $0.10 \%$ $(17,955)$ reside en Asia, principalmente en China, alcanzando el $31.3 \%(5,631 / 17,955){ }^{7}$

\section{Material y métodos}

Es un estudio observacional, descriptivo, de corte transversal, con recolección de datos retrospectiva, de los casos de IRAG reportados en el puesto centinela del Hospital Infantil de Referencia Nacional Dr. Robert Reid Cabral, Área V de salud, Distrito Nacional, desde el año 2015 hasta el año 2019. Se hace una descripción de las características de los casos ingresados a la base de datos del sistema, los virus respiratorios identificados, comportamiento en el tiempo, persona y lugar. La muestra estuvo conformada por 4,403 pacientes que fueron notificados a través del puesto centinela de Infecciones Respiratorias Agudas Graves (IRAG), del HIRRC. 
La información se tomó de la base de datos del Sistema Nacional de Vigilancia Epidemiológica (SINAVE) y fue procesada y analizada por el sustentante durante el periodo de dos meses, a través de la ficha de notificación individual digital, de la vigilancia centinela que realiza el departamento de Epidemiología del HIRRC. Esta investigación no implicó intervenciones, es decir, no hubo manipulación o contacto directo con los pacientes, por lo que esta investigación está clasificada dentro del grupo sin riesgo. Es un estudio que emplean técnicas y métodos de investigación documental retrospectivos y en los que no se realiza ninguna intervención o modificación intencionada de las variables biológicas, fisiológicas, sicológicas o sociales de los individuos que participan en el estudio.

\section{Resultados}

Tabla 1. Comportamiento epidemiológico de IRAG en el Hospital Infantil Dr. Robert Reid Cabral, 2015-2019; según año de ocurrencia

\begin{tabular}{|c|c|c|c|}
\hline Años & $\begin{array}{c}\text { Pacientes } \\
\text { con IRA }\end{array}$ & $\begin{array}{c}\text { Pacientes } \\
\text { con IRAG }\end{array}$ & $\begin{array}{c}\text { Porcen- } \\
\text { tajej de } \\
\text { IRAG/IRA }\end{array}$ \\
\hline 2015 & 11,870 & 1,706 & 14.37 \\
\hline 2016 & 13,330 & 1,350 & 10.13 \\
\hline 2017 & 12,219 & 501 & 4.10 \\
\hline 2018 & 12,580 & 465 & 3.70 \\
\hline 2019 & 11,134 & 381 & 3.42 \\
\hline Total & $\mathbf{6 1 , 1 3 3}$ & $\mathbf{4 , 4 0 3}$ & $\mathbf{7 . 2 0}$ \\
\hline
\end{tabular}

Fuente: Dirección General de Epidemiología (DIGEPI). Departamento de Epidemiología HIRRC. Sistema Nacional de Vigilancia Epidemiológica (SINAVE).
Desde el año 2015 hasta el año 2019 en la vigilancia centinela de infección respiratoria aguda grave (IRAG), se han notificado 4, 403 casos, siendo el 2015 el año con mayor número de casos reportados con un total de 1,706 casos, y en descenso en los demás años, con un total en el año 2016 de 1,350 casos, en el 2017 se reportaron 501 casos, en el 2018 se identificó 465 casos y en el 2019 se presentaron 381 casos. Según el módulo de alerta temprana del sistema nacional de vigilancia epidemiológica, desde el año 2015 hasta el año 2019 se han reportado 61,133 casos de infección respiratoria aguda (IRA), siendo el 2015 reportados un total de 11,870 casos, y siendo el 2016 el año con mayor número de casos reportados con un total de 13, 330 casos, en el 2017 se reportaron 12,219 casos, en el 2018 se identificaron 12,580 casos y en el 2019 se presentaron 11,134 casos; como se observa en el cuadro 1 , a pesar de mantenerse relativamente constante los casos de infecciones respiratorias agudas (IRA), el número de infecciones respiratorias captadas por el puesto centinela disminuyó en los años 2017, 2018 y 2019, debido, principalmente, a una modificación de la definición de casos establecida por la OPS/OMS y remitida por la Dirección General de Epidemiología. 
Gráfico 1. Comportamiento epidemiológico de IRAG en el Hospital Infantil Dr. Robert Reid Cabral, 2015-2019; según semana epidemiológica, en los años 2015- 2019

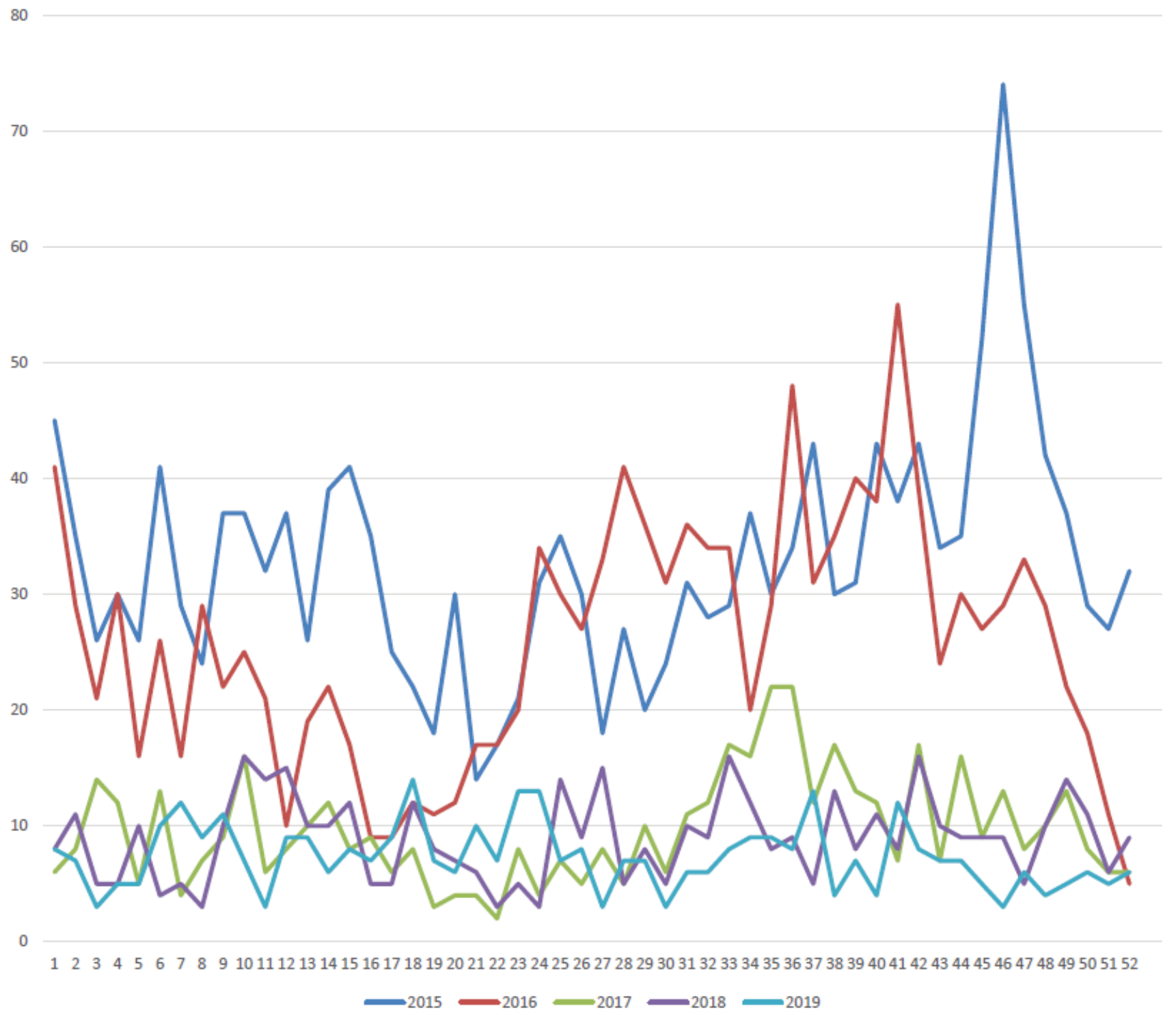

Fuente: Dirección General de Epidemiología (DIGEPI). Departamento de Epidemiología HIRRC. Sistema Nacional de Vigilancia Epidemiológica (SINAVE).

Con relación a las semanas epidemiológicas en el año 2015 hubo una mayor cantidad de casos entre las semanas 9-16 y 40-48, con un pico alto en la semana 47, en el año 2016 estuvo concentrado entre las semanas 24-42, en el año 2017, en las semanas 33-38, en el año 2018 se presentaron con mayor frecuencia entre las semanas 10-15 y con altas y bajas entre la semana 25-49, y ya para el ańo 2019 se reportaron casos de manera irregular, con altas y baja durante la semana 6-41. García Constanza, reportó en su investigación que la mayor frecuencia de casos de infección respiratoria aguda grave se detectó entre los meses de marzo a julio de 2013, con picos significativos en mayo y junio, con mayor frecuencia de muerte y con mayor circulación viral en este mismo tiempo comparada con el resto de meses del periodo de observación. ${ }^{8}$ 
Tabla 2. Comportamiento epidemiológico de IRAG en el Hospital Infantil Dr. Robert Reid Cabral, 20152019; según provincia de residencia

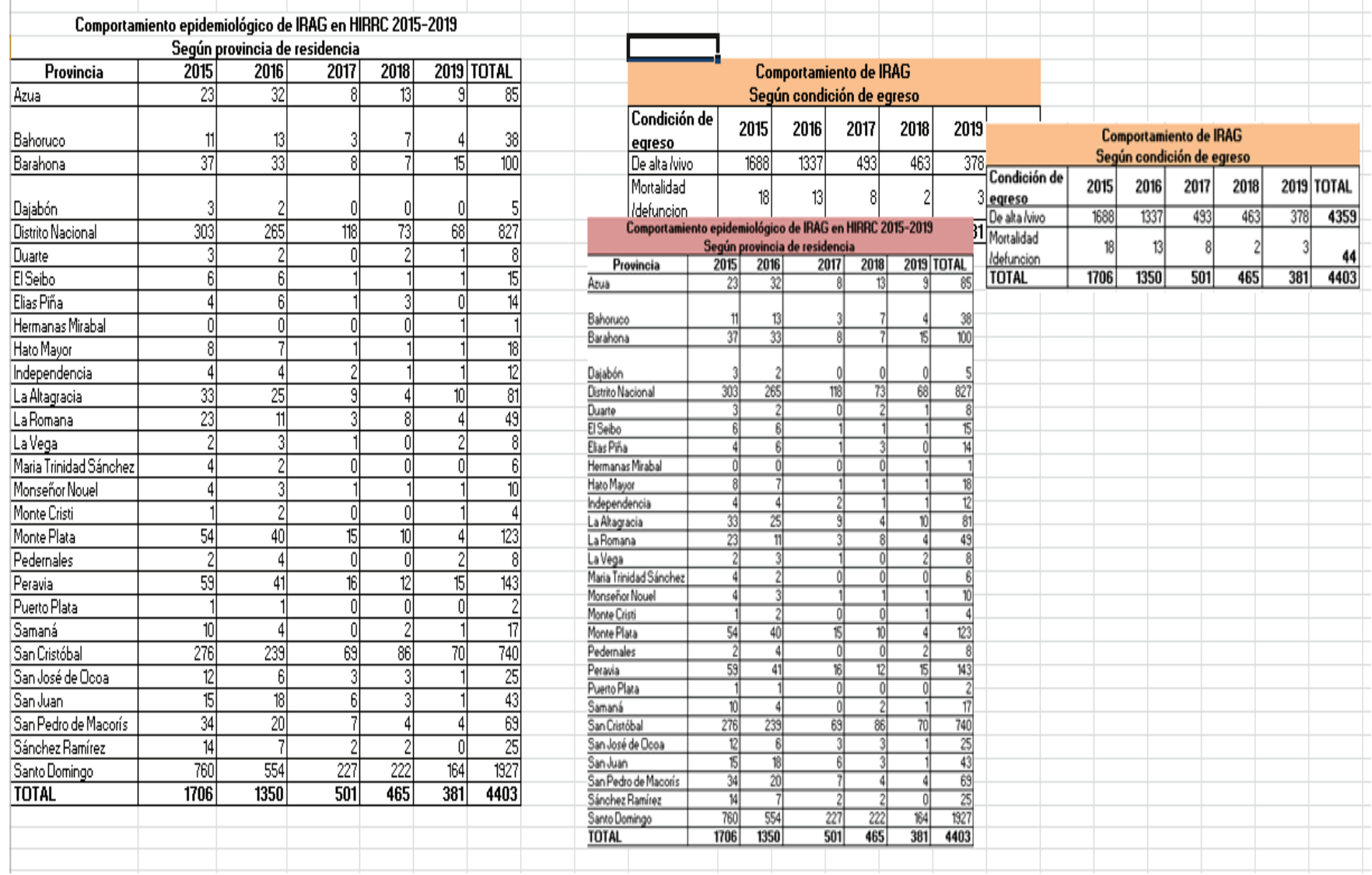

Fuente: Dirección General de Epidemiología (DIGEPI). Departamento de Epidemiología HIRRC. Sistema Nacional de Vigilancia Epidemiológica (SINAVE).

Según la provincia de residencia los tres primeros lugares en números de casos, según la provincia de residencia, estuvieron ocupados por Santo Domingo, Distrito Nacional y San Cristóbal, respectivamente, representando el $79.35 \%$ durante los cinco años estudiados. Del mismo modo Skewes-Ramm, encontró que de los casos de IRAG identificados el $70 \%$, correspondía a estas tres provincias. ${ }^{9}$ 
Gráfico 2. Comportamiento epidemiológico de IRAG en el Hospital Infantil Dr. Robert Reid Cabral, 2015-2019; según edad

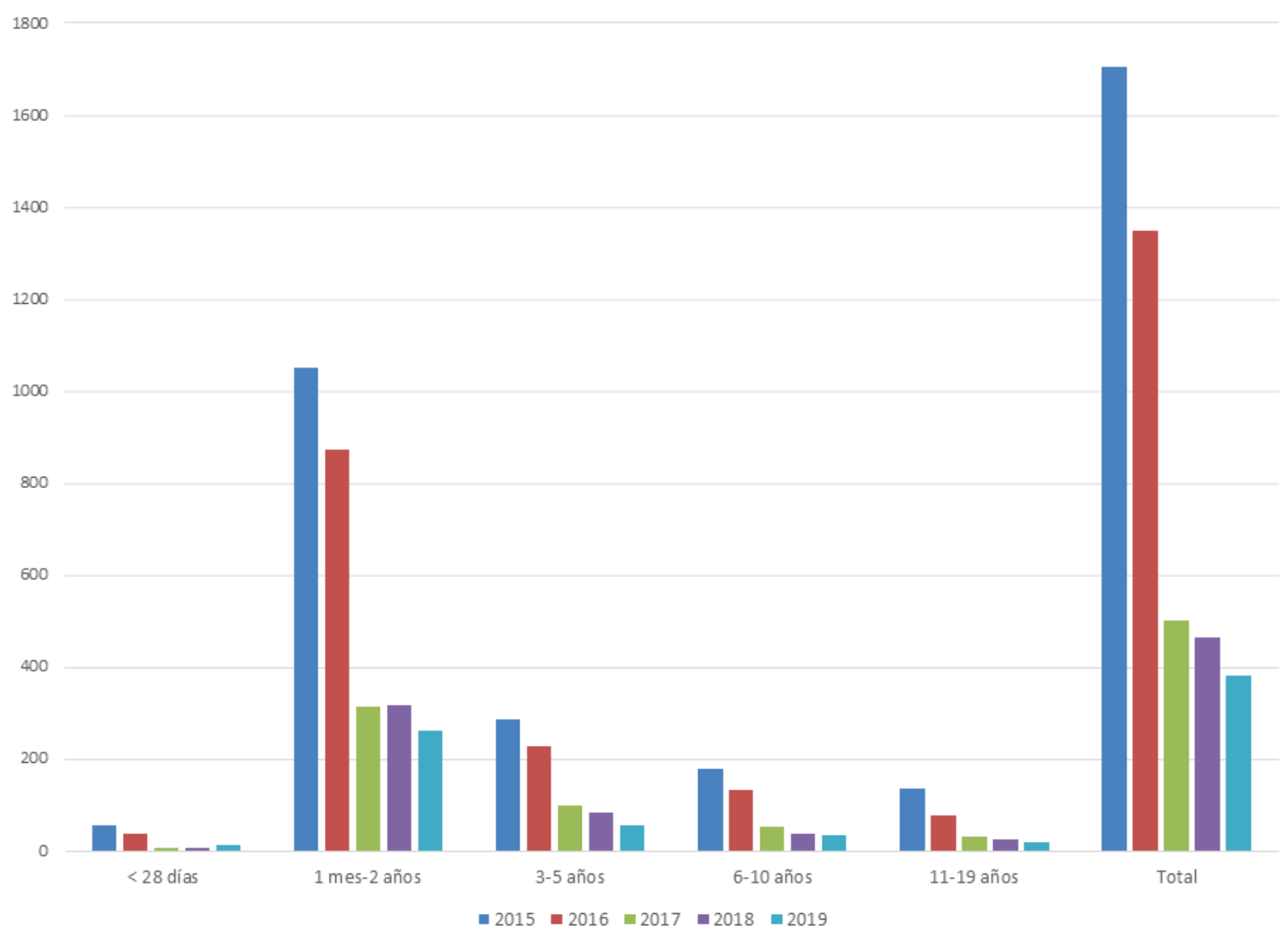

Fuente: Dirección General de Epidemiología (DIGEPI). Departamento de Epidemiología HIRRC. Sistema Nacional de Vigilancia Epidemiológica (SINAVE).

La edad en donde se reportaron más casos fue la comprendida entre el mes de nacido y los 2 ańos de edad, con un $63.88 \%$. Parecido a nuestro estudio, pero no por grupo de edad específico, sino con la media de edad de los casos en el estudio de SkewesRamm en el Hospital Infantil Dr. Robert Reid Cabral en los años 2008-2009, que fue de 2.9 años'. Con similitud a nuestro estudio, Gómez Alba Gómez Alba et al. detectaron que el grupo de edad de 0-2 meses fue el más afectado en un $74 \% .{ }^{10}$ Coincidiendo de igual forma con el estudio de García Constanza ${ }^{8}$, en el que las infecciones respiratorias agudas graves fueron más frecuentes en el grupo de edad menores de un año en el 59,5\%.

Estos resultados están en correspondencia con lo expuesto en la literatura mundial donde se plantea que en los primeros ańos de vida el niño tiene menos madurez inmunológica y menos capacidad de defensa ante infecciones. 
Tabla 3. Comportamiento epidemiológico de IRAG en el Hospital Infantil Dr. Robert Reid Cabral, 2015-2019; según sexo

\begin{tabular}{|c|c|c|c|c|c|}
\hline Sexo & $\mathbf{2 0 1 5}$ & $\mathbf{2 0 1 6}$ & $\mathbf{2 0 1 7}$ & $\mathbf{2 0 1 8}$ & $\mathbf{2 0 1 9}$ \\
\hline Masc & 998 & 802 & 280 & 273 & 199 \\
\hline Fem & 708 & 548 & 221 & 192 & 182 \\
\hline Total & $\mathbf{1 7 0 6}$ & $\mathbf{1 3 5 0}$ & $\mathbf{5 0 1}$ & $\mathbf{4 6 5}$ & $\mathbf{3 8 1}$ \\
\hline
\end{tabular}

Fuente: Dirección General de Epidemiología (DIGEPI). Departamento de Epidemiología HIRRC. Sistema Nacional de Vigilancia Epidemiológica (SINAVE).

El sexo mayor afectado en todos los años estudiados fue el masculino con un $57.96 \%$. Al igual que el estudio de García Constanza ${ }^{8}$, donde también predomino el sexo masculino en un $56 \%$. Del mismo modo Skewes- Ramm encontró que de los casos de IRAG captados por los puestos centinelas, el $57 \%$ son del sexo masculino?.

De la misma forma, Gómez Alba et al. encontraron en su investigación, al igual que nosotros, $62 \%$ de la muestra correspondió al sexo masculino. ${ }^{10}$ Publicaciones como Lagos et al. ${ }^{11}$ y Moreno Rodríguez ${ }^{12}$, plantean que el varón es más sensible a la acción de los cambios y/o alteraciones del medio ambiente, lo que los coloca en una posición desventajosa ante las infecciones.

Gráfico 3. Comportamiento epidemiológico de IRAG en el Hospital Infantil Dr. Robert Reid Cabral, 2015-2019; según virus identificado

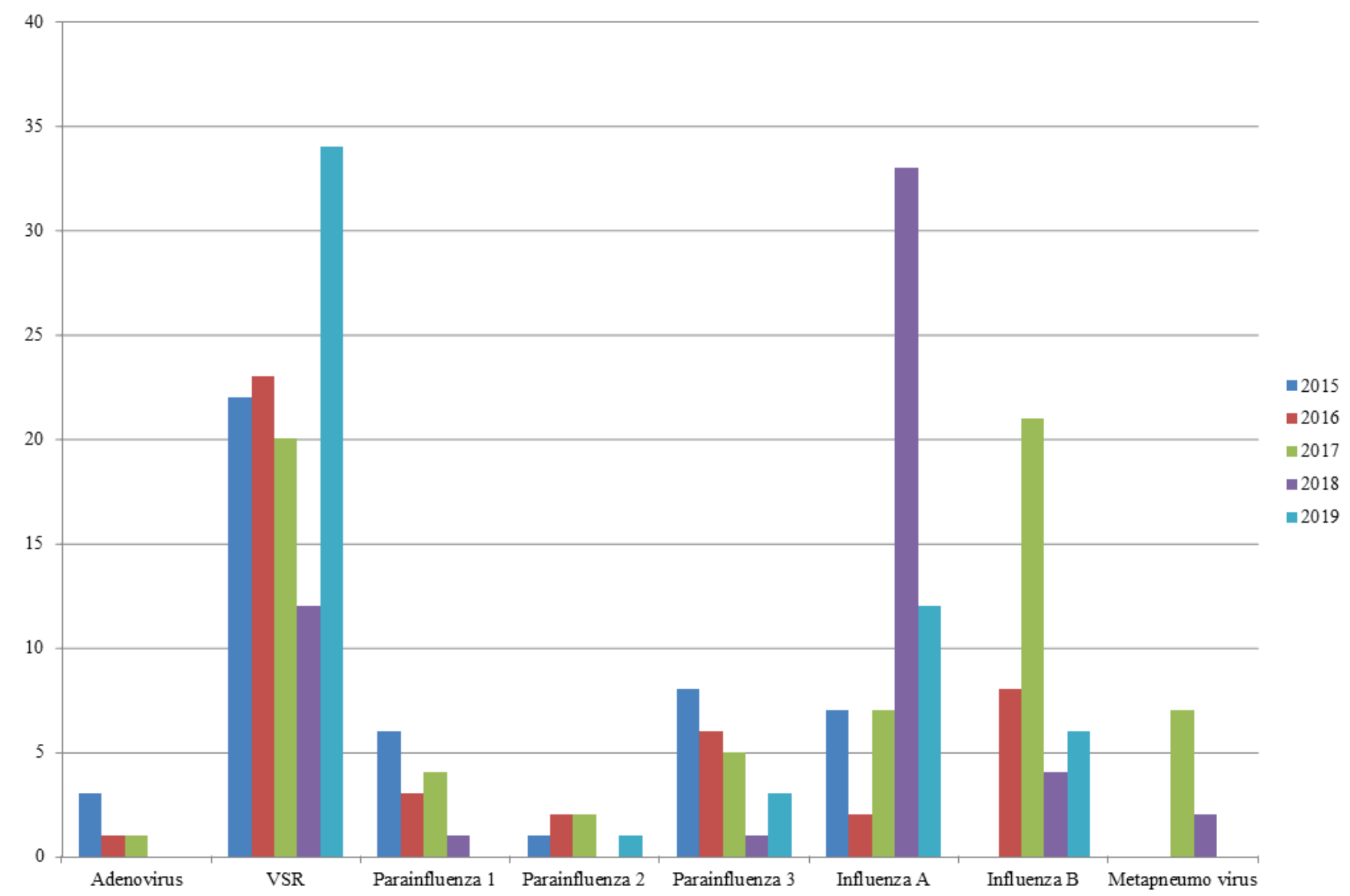

Fuente: Dirección General de Epidemiología (DIGEPI). Departamento de Epidemiología HIRRC. Sistema Nacional de Vigilancia Epidemiológica (SINAVE).

64 | Ciencia y Salud 2021; 5(1, enero-abril): 57-67 • Artículo original 
La detección de la circulación del virus sincitial respiratorio se identificó puntualmente con mayor porcentaje en los años 2015, 2016, 2017 y en el año 2019, en comparación con el año 2018 donde el agente predominante fue el virus de la influenza A H1N1. De igual forma que en nuestro estudio, García Constanza ${ }^{8}$ encontró que el virus Sincitial respiratorio causó el $75 \%$ de los casos positivos, seguido de influenza A y Adenovirus con el 11,1\%, respectivamente; Influenza $B$ el 2,7 \%, y no se registraron casos de Parainfluenza.

De la misma forma, Gómez et al. determinaron en su investigación que en el $48 \%$ se confirmó el Virus Sincitial Respiratorio (VRS) ${ }^{10}$. Mientras, diferente a nuestro estudio, Skewes-Ramm reportó que durante el año 2008-2009 circularon varios virus respiratorios, siendo el Adenovirus (34.8 \%) y Parainfluenza (34.8\%) los más frecuentes. La circulación de la Influenza A fue relativamente baja (3\%) en comparación con otros virus respiratorios. ${ }^{?}$

Tabla 4. Comportamiento epidemiológico de IRAG en el Hospital Infantil Dr. Robert Reid Cabral, 2015-2019; según condición de egreso

\begin{tabular}{|c|c|c|c|c|c|}
\hline $\begin{array}{c}\text { Condición } \\
\text { de egreso }\end{array}$ & $\mathbf{2 0 1 5}$ & $\mathbf{2 0 1 6}$ & $\mathbf{2 0 1 7}$ & $\mathbf{2 0 1 8}$ & $\mathbf{2 0 1 9}$ \\
\hline De alta/vivo & 1688 & 1337 & 493 & 463 & 378 \\
\hline $\begin{array}{c}\text { Mortalidad/ } \\
\text { defunción }\end{array}$ & 18 & 13 & 8 & 2 & 3 \\
\hline Total & $\mathbf{1 7 0 6}$ & $\mathbf{1 3 5 0}$ & $\mathbf{5 0 1}$ & $\mathbf{4 6 5}$ & $\mathbf{3 8 1}$ \\
\hline
\end{tabular}

Fuente: Dirección General de Epidemiología (DIGEPI). Departamento de Epidemiología HIRRC. Sistema Nacional de Vigilancia Epidemiológica (SINAVE).

Según la condición de egreso, de los 4403 pacientes ingresados, a 4359 se les dio de alta para un $99 \%$ y tuvimos 44 fallecidos, para un $1 \%$.

De los 4403 casos de los 5 años, 44 fallecieron para una tasa de letalidad de 9.9 por cada 1000 casos, siendo el año 2017 el que tuvo la tasa de letalidad más alta, con 16 por cada 1000 casos. Nuestra tasa de letalidad estuvo muy por debajo de la hallada por Skewes-Ramm en su estudio, quien encontró una tasa de letalidad de 30 por cada 1000 casos de IRAG. ${ }^{?}$

\section{Conclusión}

La captación de infecciones respiratorias agudas graves (IRAG), a través del puesto centinela, disminuyó en los años 2017 (501 casos), 2018 (465 casos) y 2019 (381 casos), debido, principalmente, a una modificación de la definición de casos establecida por la OPS/OMS y remitida por la Dirección General de Epidemiología, representando solo un 4.10, 3.70 y 3.42 por ciento, respectivamente, de las IRA en esos últimos tres años de estudio; sin embargo, en los dos primeros años de estudio, 2015 (1706 casos) y 2016 (1350 casos), donde no se habían disminuido los criterios de definición de caso, las IRAG representaron 14.37 y $10.13 \%$ de las IRA, respectivamente. No obstante, en las infecciones respiratorias agudas (IRA), no tenemos mucha variación de los casos anuales, siendo en el 2015 reportados un total de 11,870 casos, en el 2016 un total de 13, 330 casos, en el 2017 se reportaron 12,219 casos, en el 2018 se identificaron 12,580 casos y en el 2019 se presentaron 11,134 casos.

Con relación a las semanas epidemiológicas en el año 2015 hubo una mayor cantidad de casos entre las semanas 9-16 y 40-48, con un pico alto en la semana 47, en el año 2016 estuvo concentrado entre las semanas 24-42, en el ańo 2017, en las semanas 33-38, en el año 2018 se presentaron con mayor frecuencia entre las semanas 10-15 y con altas y bajas entre la semana 25-49 y ya para el ańo 2019 se reportaron casos de manera irregular con altas y baja durante la semana 6-41.

Según la provincia de residencia, los tres primeros lugares estuvieron ocupados por Santo Domingo, Distrito Nacional y San Cristóbal durante los cinco años estudiados. 
La edad en donde se reportaron más casos fue la comprendida entre el mes de nacido y los 2 años de edad.

El sexo más afectado en todos los años estudiados fue el masculino.

La detección de la circulación del virus sincitial respiratorio se identificó puntualmente con mayor porcentaje en los años 2015, 2016, 2017 y el año 2019, en comparación con el año 2018 donde el agente predominante fue el virus de la influenza $A$ H1N1. Según la Organización Mundial de la Salud, el Virus Sincitial Respiratorio (VSR) y el virus tipo 3 de la Parainfluenza son las causas principales de infecciones respiratorias agudas en la infancia y la niñez temprana, causando del 20 al $25 \%$ de los casos de neumonía y del $45 \%$ al $50 \%$ de bronquiolitis en niños hospitalizados. ${ }^{13}$

Según la condición de egreso, de los 4403 pacientes ingresados, a 4359 se les dio de alta para un $99 \%$ y tuvimos 44 fallecidos, para un $1 \%$. De los 4403 casos de los cinco años, 44 fallecieron para una tasa de letalidad de 9.9 por cada 1000 casos, siendo el año 2017 el que tuvo la tasa de letalidad más alta, con 16 por cada 1000 casos.

El propósito de la vigilancia intensificada es contribuir con la formulación de estrategias de intervención para la prevención y el control y de infecciones respiratorias agudas graves a nivel local, sub nacional y nacional. ${ }^{14}$

\section{Recomendaciones}

Aumentar los insumos para la toma de muestra, actualmente solo tenemos una cuota límite de 5 muestras semanales, de esta forma se elevaría el porcentaje de caracterización de los virus respiratorios.

Reportar más rápidamente los resultados de las muestras tomadas y enviadas, para retroalimentar en más corto tiempo el record clínico del paciente y al médico clínico tratante.
Incentivar líneas de investigación que incluyan características clínicas, además de epidemiológicas, para tener una visión más integral de la situación integral de las infecciones respiratorias agudas graves.

Aumentar a través de las Direcciones Provinciales de Salud (DPS) y las Direcciones de Áreas de Salud (DAS) la promoción de hábitos saludables, incentivando factores de riesgo modificables para evitar o disminuir el desarrollo de las infecciones respiratorias agudas (IRA) y las infecciones respiratorias agudas graves (IRAG), facilitar educación a las madres, padres o cuidadores durante las consultas, de forma que se propicie que estos obtengan los conocimientos que permitan la adopción de actitudes y prácticas protectoras para la salud de los niños.

\section{Bibliografía}

1. Sánchez N. Reporte Técnico de la Vigilancia, V.1 octubre 1999 ISSN 1028-4338.

2. Organización Mundial de la Salud. Plan de acción estratégico de la OMS para la gripe pandémica. Ginebra: OMS; 2006.

3. Instituto Nacional de Salud (Colombia). Informe evento Infección Respiratoria Aguda, periodo epidemiológico 12. Bogotá: INS; 2012.

4. Instituto Nacional de Salud (Colombia). Protocolo de vigilancia y control de infección respiratoria aguda. Bogotá: INS; 2012.

5. Ministerio de Salud Pública. Subsistema de Vigilancia de enfermedades emergentes y reemergentes. Protocolo de Vigilancia de infección respiratoria aguda. República Dominicana. Febrero 2020.

6. Speranza AM, Clary AL, Pereira T, Zaposnikoff L, Schenone N. Estudio multicéntrico de infecciones respiratorias agudas bajas en niños hospitalizados menores de dos años. Arch. argent pediatr, 2003;101(6):365-74, 
7. Ministerio de Salud Pública y Asistencia Social. Preparativos frente al riesgo de introducción del nuevo Coronavirus (2019-nCoV) al territorio dominicano - 23 de enero del 2020.

8. García Constanza, M. Caracterización epidemiológica de la infección respiratoria aguda grave y circulación viral en Boyacá, Colombia, julio de 2012 a julio de 2013.

9. Skewes-Ramm R. Análisis del sistema de vigilancia centinela de las Infecciones Respiratorias Agudas Graves, República Dominicana; 2010.

10. Gómez Alba V, Feris-Iglesias J, Florén A, et al. Características clínico-epidemiológico de la infección respiratoria aguda (IRA) por Virus Sincitial Respiratorio (VSR) en niños menores de dos años: admitidos en el hospital Infantil Dr. Robert Reid Cabral (HIRRC), enero-diciembre 2006.

11. Lagos ZR, Avendaño CLF, Levine MM. Vigilancia sistemática de virus influenza, respiratorio sincitial, parainfluenza y adenovirus en niños ambulatorios con infecciones respiratorias agudas. Rev Med Chile. 1999 Sep; 127(9)1063-72. Disponible en: https://scielo.conicyt.cl/scielo.php?script=sci_ arttext\&pid=S0034-98871999000900005\& lng=es. http://dx.doi.org/10.4067/S0034-9887 1999000900005.
12. Moreno Rodríguez LL. Infecciones Respiratorias Agudas en niños menores de 5 años [Internet]. 2009 [Aragua, Venezuela] [aprox. 7p.] Disponible en: ISSN 1028-9933 $399 \mathrm{http}: / /$ www. portalesmedicos.com/publicaciones/articles/1622/1/Infecciones-Respiratorias-Agudas-en-ninos-menores-de-5-anos.htm

13. Organización Panamericana de la Salud. Oficina Regional de la Organización Mundial de la Salud. Guía operativa para la vigilancia nacional intensificada de Infección Respiratoria Aguda Grave IRAG. Proyecto de prevención y control de enfermedades transmisibles. Washington D.C. OPS; 2011.

14. Instituto Nacional de Salud (Colombia). Informe evento Infección Respiratoria Aguda, periodo epidemiológico 12. Bogotá: INS; 2012.

15. DIGEPI [Internet]. Morbilidad de Enfermedades de Notificación Obligatoria en Hospital Infantil Dr. Robert Reid Cabral. 2018-2019. Disponible en: http://digepisalud.gob.do/ 